\title{
OCORRÊNCIA DE SARASINULA MARGINATA (MOLLUSCA: SOLEOLIFERA) EM BELO HORIZONTE, MG, BRASIL*
}

Lads Clark Lima**

Cecilia Pereira de Souza**

\begin{abstract}
LIMA, L.C. \& SOUZA, C.P. de [Occurrence of Sarasınula margınata (Mollusca: Soleolifera) in Belo Horiozonte, MG, Brazill. Rev. Saúde públ., S. Paulo, 23:345-6, 1989.
\end{abstract}

\begin{abstract}
The occurrence of Sarasinula marginata, a possible intermediate host of Anglostrongylus costaricensis, in the city of Belo Horizonte, MG, Brazil, is reported on.
\end{abstract}

KEYWORDS: Sarasinula margtnata, parasitology, Nematode infections, occurrence.

Várias espécies de lesmas da família Veronicellidae são hospedeiras intermediárias do $A n$ giostrongylus costaricensis Morera e Céspedes, 1971 (Nematoda: Metastrongylidae). No Brasil, casos humanos de angiostrongiliase abdominal têm sido registrados nos Estados de São Paulo, Paraná, Santa Catarina e Rio Grande do Sul, além do Distrito Federal (Graeff-Teixeira e col.1, 1987).

No Rio Grande do Sul, a Phyllocaulis variegatus (Semper, 1885) é hospedeira intermediária do referido parasita, e a Phyllocaulis soleiformes (Orbigny, 1835) mostrou-se suscetível ao mesmo em infestação experimental (Teixeira e col.3, 1988). Por outro lado, na Costa Rica, a Sarasinula plebeia (Fischer, 1868) é transmissora do $A$. costaricensis (Morera e Ash2, 1970).

No Brasil, espécies do gênero Sarasinula são encontradas desde o Rio Grande do Sul até o Amazonas (Thomé4, 1975); todavia, no Estado de Minas Gerais somente encontramos o regis- tro de ocorrência deste gênero na cidade de Juiz de Fora (Thomé4, 1975).

Estes fatos levaram-nos a registrar a ocorrência de espécimes de Sarasinula em jardim residencial da cidade de Belo Horizonte, Minas Gerais, em novembro e dezembro de 1988.

Os exemplares foram fixados pela técnica de Thomés (1975) e identificados como Sarasinula marginata (Semper, 1885) (Fig. A e B). Foram incluídos na coleção da Fundação Zoobotânica do Rio Grande do Sul, sob o número MCN3188.

Acreditamos que tentativas de encontrar estas lesmas naturalmente infestadas com $A$. costaricensis devem ser feitas, bem como de infestá-las experimentalmente.

\section{AGRADECIMENTO}

Ao Dr. José Willibaldo Thomé, pela identificação dos moluscos.
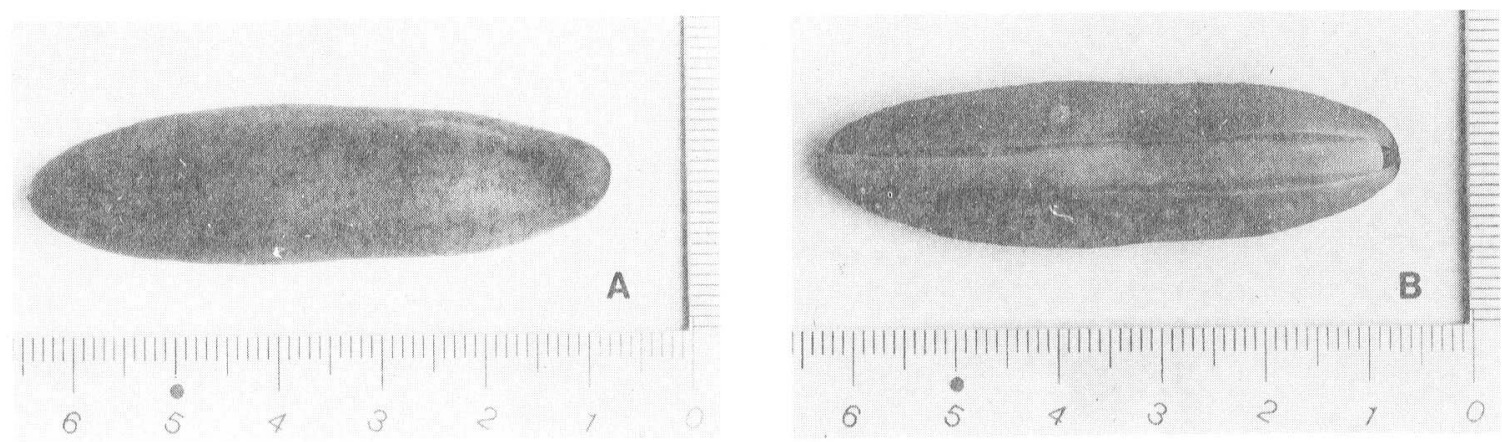

Fig.: Sarasmula marzmala. A: vista dirsal. B: vesta venllal.

* Trabalho parcialmente financiado nela Financiadora de Estudos e Projctos (FINEP) - Processo n: $1282: 88$.

* Laboratório de Molacolugia do Centro de Pesquisas René Rachou. Fundaçáo Oswaldo (rur (FIOCRUZ) -.. (alxa Postal 1743 - 30190 -.. Helo Horizonte, MG - Brasil. 


\section{REFERÊNCIAS BIBLIOGRÁFICAS}

1. GRAEFF-TEIXEIRA, $C$. et al. Abdominal angiostrongyliasis - an underdiagnosed disease. Mem. Inst. Oswaldo Cruz, Rio de Janeiro, 82(suppl.):3534, 1987.

2. MORERA, P.\& ASH, L.R. Investigación del huésped intermediario de Angiostrongylus costaricensis Morera y Céspedes, 1971. Bol. chil. Parasitol., 25:135, 1970.

3. TEIXEIRA, G.C. et al. Suscetibilidade da lesma Phyllocaulis soleiformis à infecção por Angiostrongylus costaricensis. Rev. Soc. bras. Med. trop. S. Paulo, 21 (supl.):94, 1988.
4. THOMÉ, J.W. Os gêneros da família Veronicellidae nas Américas (Mollusca: Gastropoda). IheringiaZool., 48:3-56, 1975.

5. THOMÉ, J.W. Distensão de moluscos terrestres para fixação, com comentários sobre coleta e transporte. Arq. Mus. Nac., Rio de Janeiro, 55:153-4,1975.

Recebido para publicação em 6/7/1989 Aprovado para publicação em $13 / 7 / 89$ 